\section{Adherencia a las recomendaciones de tromboprofilaxis post-cesárea: estudio de corte transversal}

\author{
SOFÍA GRILLE ${ }^{1}$, LUANA MAGGIARI ${ }^{\mathrm{a}}$, IGNACIO MORALES ${ }^{\mathrm{a}}$, \\ JONATHAN OJEDA ${ }^{\text {a }}$, BRIAN PÉREZ ${ }^{\text {a }}$ IGNACIO PÉREZ ${ }^{a}$, \\ VIRGINIA RIBERO ${ }^{a}$, CECILIA GUILLERMO $^{1, a}$
}

\section{Compliance with post-cesarean section thromboprophylaxis recommendations. A cross sectional study in 233 women}

Background: Cesarean section increases four times the risk of venous thromboembolism compared to vaginal delivery. The Royal College of Obstetricians and Gynecologists guidelines are used at our service. A written alert was designed to stratify patients at high, intermediate or low risk making a suggestion for thromboprophylaxis. Aim: To assess the compliance with the guidelines and to evaluate the impact of a written alert in the thromboprophylaxis compliance in women subjected to caesarean section. Patients and Methods: Review of medical records of 233 women aged 19 to 32 years, subjected to a caesarean section in a Gynecology Service, between 2016-2017. Results: Compliance with recommendations was observed in 29\% of patients (68/233), 86\% in the low-risk group, $26 \%$ in the intermediate risk group and 100\% in the high risk group. In 41/233 (18\%) of patients, a written alert was included in the medical record. Compliance with recommendations in the presence of the written alert was 61\% (25/41 women) compared to $22 \%(43 / 192)$ in those lacking the alert $(p<0.01)$. In women whose emergency caesarean section was the only risk factor, the compliance with the recommendation was $8 \%$, compared with $30 \%$ among those who had at least one thrombotic risk factor associated with caesarean section $(p<0.01)$. Conclusions: In this cross-sectional study, we observed a low compliance with thromboprophylaxis guidelines in cesarean women. We observed that the use of a written alert improved the compliance with thromboprophylaxis.

(Rev Med Chile 2021; 149: 881-887)

Key words: Cesarean Section; Pregnancy; Reference Standards; Thrombosis.

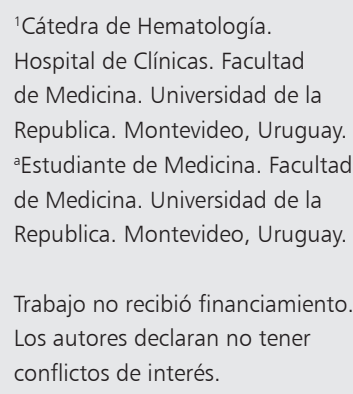

Recibido el 16 de abril de 2020, aceptado el 19 de abril de 2021

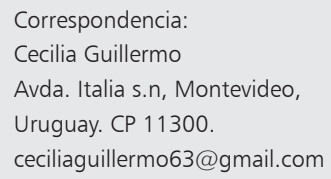

L a tromboembolia venosa (TEV) ocurre aproximadamente en 1,2 de cada 1.000 partos $^{1,2}$. A pesar del bajo riesgo absoluto que presenta, es una de las principales causas de morbi-mortalidad materna ${ }^{3}$. Además la trombosis venosa profunda (TVP) relacionada a la gestación tiene una elevada tasa de síndrome post-trombótico con el consiguiente deterioro de la calidad de vida que ello implica ${ }^{4}$.
La utilización de tromboprofilaxis durante el embarazo y puerperio es un tema de gran controversia. No hay ensayos clínicos randomizados que evalúen la tromboprofilaxis en el embarazo o puerperio dado que los mismos han sido difíciles de implementar ${ }^{5}$. La mayoría de las recomendaciones se basan en estudios retrospectivos y en la opinión de expertos con un nivel de evidencia bajo.

La cesárea es una de las operaciones quirúrgicas 
más frecuentes del mundo, con tasas que siguen subiendo, en particular en los países de ingresos medios y altos con elevada accesibilidad a la misma. En Latinoamérica la tasa de cesárea variaba entre $3,0 \%$ a $45,9 \%{ }^{6}$. A modo de ejemplo, en Brasil es de 45,9\%, México 37,8\%, Argentina 35,2\%, Uruguay 31,9\%, Chile 30,7\%, Haití 3,0\%, entre otros $^{6}$. En la mayoría de los estudios publicados, el parto por cesárea se asocia con mayor tasa de complicaciones trombóticas que el parto vaginal ${ }^{7}$. Un meta-análisis publicado en 2016 mostró que la cesárea aumenta 4 veces el riesgo de VTE en forma independiente con otros factores de riesgo comparado con parto vaginal. Este riesgo es mayor para la cesárea de emergencia. Aproximadamente 3 de cada 1.000 mujeres desarrollarán una TVP luego de una césarea ${ }^{8}$.

Si bien existen diferencias en cuanto a las recomendaciones de realizar tromboprofilaxis en nuestro hospital hemos consensuado seguir las guías del Royal College of Obstetricians and Gynaecologists (RCOG) ${ }^{9}$. Para ello se diseñó una alerta escrita que estratifica el riesgo y sugiere la indicación de tromboprofilaxis farmacológica. Nuestro primer trabajo evaluando la implementación de las guías mostró que el grupo donde los médicos adherían menos era en las mujeres que se les realizaba una cesárea ${ }^{10}$. Asimismo, tuvimos dificultades en la implementación universal de la alerta. En base a esto, el objetivo de este trabajo fue evaluar en la población de mujeres que se les realizó cesárea la adherencia a la pauta de tromboprofilaxis y el alcance e impacto que tiene la alerta escrita.

\section{Pacientes y Métodos}

Estudio transversal donde se incluyeron todas las mujeres mayores de 18 años a las que se les realizó una cesárea de coordinación o de emergencia en el Servicio de Ginecotología del Hospital de Clínicas, Montevideo-Uruguay, desde enero/2016-enero/2017. Se excluyeron del estudio las mujeres que recibieron anticoagulación durante el embarazo por otra indicación.

Se incluyeron 233 mujeres con una mediana de edad de 23 años (19-32). En la Tabla 1 se muestra las características de la población.

Se revisaron las historias clínicas y los datos clínicos fueron recabados. Se registraron los factores de riesgo descritos por la RCOG y las pacientes fueron clasificadas en: alto, intermedio y bajo riesgo ${ }^{9}$. La guía hace sugerencias en cuanto a la dosis de heparina de bajo peso molecular (HBPM) a usar en relación al peso de las pacientes. Se registró si recibieron la tromboprofilaxis farmacológica sugerida por la guía y si existió un evento trombótico o hemorrágico. Adicionalmente se registró si el equipo que lo asistió contaba en la historia clínica con la alerta escrita para guiar la tromboprofilaxis o no.

Se realizó un análisis descriptivo de los datos utilizando medidas de resumen y dispersión. Para el análisis de las diferencias entre grupos se utilizó el test estadístico de chi cuadrado y el test no paramétrico de Mann Whitney para las variables continuas. Se consideró significativo un valor de $\mathrm{p}<0,05$. Para el procesamiento de las variables en estudio, se empleó el paquete estadístico SPSS versión 20.0.

El estudio fue aprobado por el Comité de Ética del Hospital de Clínicas.

\section{Resultados}

En la Tabla 1 se muestra las características de la población. Se destaca que $14,6 \%$ de las mujeres tenían más de 35 años, 36,5\% eran tabaquistas, $11,1 \%$ tenían un índice de masa corporal (IMC) mayor a $30 \mathrm{~kg} / \mathrm{m}^{2}$ y $12,0 \%$ presentaron pre-eclampsia. De las cesáreas, 59,6\% fueron de emergencia.

De las 233 mujeres incluidas, en 41 (17,6\%) se incluyó en la historia clínica una alerta escrita que le facilitaba y recordaba al equipo de salud realizar la estratificación de riesgo trombótico para guiar la tromboprofilaxis farmacológica.

Según el sistema de estratificación de riesgo de la RCOG $^{9}$ las mujeres fueron clasificadas en 3 grupos: riesgo bajo, riesgo intermedio y alto riesgo. En la Tabla 2 se muestran los datos de la población estratificada por grupos de riesgo.

En cuanto a la adherencia a las guías del RCOG, la misma se consiguió en 29,2\% (68 mujeres). Si tomamos en cuenta la adherencia por grupo de riesgo: $85,7 \%$ (6/7 mujeres) en el grupo de bajo riesgo, $25,5 \%$ (56/220 mujeres) en el grupo de riesgo intermedio y $100 \%$ de adherencia (6/6 mujeres) en el grupo de alto riesgo. En el grupo de bajo riesgo una paciente recibió tromboprofilaxis 
Tabla 1. Características de la Población

\begin{tabular}{|lc|}
\hline Variables & $\mathbf{n}=\mathbf{2 3 3}$ \\
\hline Edad (mediana, RIQ) & $23(19-32)$ \\
\hline Edad > 35 años & $14,6 \%$ \\
\hline Semanas de edad gestacional (mediana, RIQ) & $38(35-40)$ \\
\hline Cesárea emergencia & $59,6 \%$ \\
\hline Cesárea de coordinación & $40,3 \%$ \\
Comorbilidades médicas & $6,4 \%$ \\
\hline Antecedente de trombosis & $1,7 \%$ \\
\hline Trombofilia asintomática & $0,86 \%$ \\
\hline Procedimientos quirúrgicos & $0,43 \%$ \\
\hline IMC > 30 kg/m² & $11,1 \%$ \\
\hline Tabaquismo & $36,5 \%$ \\
Paridad & $2(1-3)$ \\
\hline Gruesas venas varicosas & $1,7 \%$ \\
\hline Infección sistémica en puerperio & $3 \%$ \\
\hline Inmovilidad & $3 \%$ \\
\hline Pre-eclampsia & $12,0 \%$ \\
\hline Trabajo de parto prolongado & \\
Hemorragia postparto con pérdida de más & $1,3 \%$ \\
de 1 litro o requerimiento transfusional & \\
\hline
\end{tabular}

RIQ: rango intercuartil; IMC: índice de masa corporal. y el análisis de la historia clínica no reveló cual fue su indicación. Dentro del grupo intermedio 4 pacientes presentaban contraindicaciones para la tromboprofilaxis dado por sangrado activo o por plaquetopenia y esto se tuvo en cuenta a la hora de evaluar la adherencia a las recomendaciones.

Al evaluar el impacto de la presencia de la alerta escrita en la historia clínica observamos que en las mujeres que tenían una alerta escrita en la historia al momento de indicar la tromboprofilaxis, en $60,9 \%$ (25/41 mujeres) se había seguido la guía de tromboprofilaxis en forma adecuada mientras que en las mujeres que no contaban con dicha alerta escrita la adherencia a la guía fue de $22,4 \%$ (43/192), $p=0,0001$.

En la Tabla 3 se muestran los resultados del grupo de riesgo intermedio (94,4\% de las mujeres) en cuanto a la adherencia a la tromboprofilaxis según los factores de riesgo. Dentro de ellos la adherencia a la tromboprofilaxis en mujeres con un IMC $>40$ fue de $50 \%$, con comorbilidades médicas fue de $35,7 \%$, con cesárea de coordinación fue de $28 \%$, con cesárea de emergencia fue de $24,5 \%$ y de las que desarrollaron pre-eclampsia fue de $40,7 \%$.

Tabla 2. Características de la población por grupo de riesgo trombótico

\begin{tabular}{|c|c|c|c|}
\hline Variables & $\begin{array}{c}\text { Alto riesgo } \\
(n=6)\end{array}$ & $\begin{array}{l}\text { Riesgo intermedio } \\
(n=220)\end{array}$ & $\begin{array}{c}\text { Bajo riesgo } \\
\quad(n=7)\end{array}$ \\
\hline Edad (mediana \pm RIQ) & $35 \pm 7$ & $25 \pm 8$ & $26 \pm 8$ \\
\hline Edad > 35 años (n/\%) & $4 / 66,7 \%$ & $30 / 13,6 \%$ & 0 \\
\hline Semanas de edad gestacional (mediana $\pm \mathrm{RIQ}$ ) & $39 \pm 3$ & $38 \pm 3$ & $37 \pm 4$ \\
\hline Cesárea emergencia (n/\%) & $2 / 33,3 \%$ & $137 / 62,3 \%$ & 0 \\
\hline Cesárea de coordinación (n/\%) & $4 / 66,7 \%$ & $82 / 37,3 \%$ & $7 / 100 \%$ \\
\hline Comorbilidades médicas (n/\%) & $1 / 16,7 \%$ & $14 / 6,4 \%$ & 0 \\
\hline Antecedente de trombosis (n/\%) & 0 & $4(1,8 \%)$ & 0 \\
\hline Trombofilia asintomática (n/\%) & $2(33,3 \%)$ & 0 & 0 \\
\hline Procedimientos quirúrgicos (n/\%) & 0 & $1(0,5 \%)$ & 0 \\
\hline $\mathrm{IMC}>30 \mathrm{~kg} / \mathrm{m}^{2}(\mathrm{n} / \%)$ & $1(33,3 \%)$ & $25(14,6)$ & 0 \\
\hline Tabaquismo (n/\%) & $4(66,7 \%)$ & $80(36,4 \%)$ & $1(14,3 \%)$ \\
\hline Paridad (n/\%) & $4(3-5)$ & $2(1-3)$ & $2(1-3)$ \\
\hline Gruesas venas varicosas (n/\%) & $1(16,7 \%)$ & $3(1,4 \%)$ & 0 \\
\hline Infección sistémica en puerperio & $1(16,7 \%)$ & $6(2,7 \%)$ & 0 \\
\hline Inmovilidad & 0 & $7(3,2 \%)$ & 0 \\
\hline Pre-eclampsia & $1(16,7 \%)$ & $27(12,3 \%)$ & 0 \\
\hline \multirow{2}{*}{$\begin{array}{l}\text { Trabajo de parto prolongado } \\
\text { Hemorragia postparto con pérdida de más de } 1 \text { litro o } \\
\text { requerimiento transfusional }\end{array}$} & 0 & $16(7,3 \%)$ & 0 \\
\hline & 0 & $3(1,3 \%)$ & 0 \\
\hline
\end{tabular}

RIQ: rango intercuartil; IMC: índice de masa corporal. 
Tabla 3. Adherencia a las guías de RCOG en grupo de riesgo intermedio según factores de riesgo

\begin{tabular}{|c|c|c|c|}
\hline Variables & $\begin{array}{l}\text { Adherencia a } \\
\text { tromboprofilaxis }\end{array}$ & $\begin{array}{l}\text { No adherencia a } \\
\text { tromboprofilaxis }\end{array}$ & Total \\
\hline Edad > 35 años (n/\%) & $11(36,7 \%)$ & $19(63,3 \%)$ & 30 \\
\hline $\mathrm{IMC}>40 \mathrm{~kg} / \mathrm{m}^{2}(\mathrm{n} / \%)$ & $2(50 \%)$ & $2(50 \%)$ & 4 \\
\hline Paridad $>3$ & $26(34,2 \%)$ & $50(65,8 \%)$ & 76 \\
\hline Tabaquismo & $16(20 \%)$ & $64(80 \%)$ & 80 \\
\hline Comorbilidades Medicas & $5(35,7 \%)$ & $9(64,3 \%)$ & 14 \\
\hline Cesárea de coordinación & $23(28 \%)$ & $59(72 \%)$ & 82 \\
\hline Cesárea de emergencia & $33(24,1 \%)$ & $104(75,9 \%)$ & 137 \\
\hline Infección sistémica en puerperio & $3(50 \%)$ & $3(50 \%)$ & 6 \\
\hline Inmovilidad & $5(71,4 \%)$ & $2(28,6 \%)$ & 7 \\
\hline Pre-eclampsia & $11(40,7 \%)$ & $16(59,3 \%)$ & 27 \\
\hline Embarazo múltiple & $1(20 \%)$ & $4(80 \%)$ & 5 \\
\hline Trabajo de parto prolongado & $4(25 \%)$ & $12(75 \%)$ & 16 \\
\hline $\begin{array}{l}\text { Hemorragia postparto con pérdida de más de } 1 \text { litro o } \\
\text { requerimiento transfusional }\end{array}$ & $1(33,3 \%)$ & $2(66,7 \%)$ & 3 \\
\hline Procedimientos quirúrgicos & $1(100 \%)$ & $0(0 \%)$ & 1 \\
\hline Internación prolongada & $33(20 \%)$ & $132(80 \%)$ & 165 \\
\hline
\end{tabular}

IMC: índice de masa corporal.

Si analizamos las mujeres a las que se les realizó una cesárea de emergencia $(n=139)$, en $25,9 \%$ de éstas (36/139 mujeres), la cesárea era el único factor de riesgo trombótico que presentaban. En ellas la adherencia a la tromboprofilaxis fue de $8,3 \%$ (3/36 mujeres) mientras que en las que tenías al menos 1 factor de riesgo trombótico asociado a la cesárea fue de 30,1\% (31/103 mujeres), $\mathrm{p}=0,004$. Dentro de los casos que adhirieron a la tromboprofilaxis, $100 \%$ tenían la alerta escrita en la historia.

En cuanto a la adherencia a la dosis de HBPM utilizada en las pacientes que se les indicó tromboprofilaxis, $79 \%(47 / 59)$ recibieron la dosis adecuada. Los errores se dieron en 6 pacientes con menos de $50 \mathrm{~kg}$ que recibieron enoxaparina $40 \mathrm{mg} /$ día, 1 paciente que pesaba entre 50 y $90 \mathrm{~kg}$ que recibió enoxaparina $60 \mathrm{mg} /$ día y 5 pacientes con pesos superiores a $90 \mathrm{~kg}$ que recibieron $40 \mathrm{mg} /$ día.

En la población estudiada no se registraron eventos hemorrágicos vinculados a la administración de tromboprofilaxis farmacológica ni eventos trombóticos.

\section{Discusión}

La cesárea aumenta 4 veces el riesgo de TEV comparado con el parto vaginal, siendo mayor en la cesárea de emergencia ${ }^{8,11}$. En un trabajo retrospectivo llevado a cabo en Estados Unidos se recabaron datos de 1.461.270 embarazos que finalizaron en cesárea y se registró 6,5 muertes maternas/100.000 embarazos donde la embolia pulmonar explicó $9 \%$ de dichas muertes ${ }^{12}$. Si extrapolamos datos de la población adulta no embarazada, la tromboprofilaxis farmacológica o mecánica es capaz de reducir en $70 \%$ la incidencia de TEV ${ }^{13-16}$. No contamos con estos datos en la población embarazada, pero no hay razón para pensar que no sea efectiva.

Está ampliamente consensuado que las pacientes con alto riesgo de desarrollar una TEV durante el embarazo/puerperio deben recibir profilaxis farmacológica. Sin embargo, la tromboprofilaxis ya sea mecánica o farmacológica luego de una cesárea es un hecho en discusión. En 2011 el American Congress of Obstetricians and Gynecologists (ACOG) 
sugieren en sus guías el uso rutinario de compresión neumática intermitente en el perioperatorio de la cesárea ${ }^{17}$. La tromboprofilaxis farmacológica puede aportar beneficio adicional en mujeres en alto riesgo trombótico como es la cesárea. En este sentido, las guías varían en sus sugerencias, las guías del ACOG recomiendan profilaxis con HBPM o heparina no fraccionada (HNF) para mujeres sometidas a cesárea con trombofilias de alto riesgo, historia previa de TVE y/o historia familiar de TVE y trombofilia ${ }^{17}$. Las guías del American College of Chest Physicians (Chest) recomiendan profilaxis con HBPM para mujeres que tienen un factor de riesgo mayor o 2 menores asociados, siendo considerada la cesárea de emergencia un factor de riesgo menor. La cesárea de coordinación no se considera de riesgo. Recomienda la utilización de profilaxis mecánica cuando existe contraindicación para la utilización de $\mathrm{HBPM}^{18}$. Las guías del RCOG recomiendan profilaxis farmacológica en pacientes con cesárea de emergencia y en las de coordinación que asocian otros factores de riesgo?.

A pesar de las controversias en las indicaciones de tromboprofilaxis, nuestro servicio ha incorporado la utilización de las guías de RCOG con resultados favorables en los pacientes en los cuales se les incluye la alerta escrita ${ }^{10}$. Nuestros resultados previos, advertían que en la población de mujeres sometidas a cesárea, el equipo de salud adhería menos al seguimiento de la guía que en otras situaciones clínicas ${ }^{10}$. En este trabajo transversal que incluye todas las mujeres a las que se le realizó una cesárea, la adherencia a las guías del RCOG se consiguió en una baja proporción de casos, 29,2\% de las mujeres. En quienes se realizó tromboprofilaxis la dosis de HBPM usada fue correcta en 79\% de los casos, teniendo dificultades en mujeres con sobrepeso/obesidad probablemente vinculada al temor al sangrado con dosis superiores de HBPM. Es de destacar que en las guías utilizadas la cesárea de emergencia es considerado de riesgo intermedio y por tanto se recomienda la utilización de HBPM. En nuestra población en 59,6\% de los casos las cesáreas fueron de emergencia.

Particularmente, la adherencia a la tromboprofilaxis en mujeres que presentan cesárea de emergencia como único factor de riesgo para ser catalogadas como de riesgo intermedio y requerir tromboprofilaxis fue muy baja $(8,3 \%)$ en relación a las mujeres que además de haberse realizado una cesárea de emergencia presentaban factores de riesgo adicionales $(30,1 \%), p=0,004$. Dentro de los casos que adhirieron a la tromboprofilaxis, $100 \%$ tenían la alerta escrita en la historia. Estos resultados sugieren que los médicos ginecotólogos no consideran que la cesárea de emergencia determine suficiente riesgo trombótico que justifique la realización de tromboprofilaxis farmacológica en forma sistemática.

Esta baja adherencia a la utilización de HBPM en el post-operatorio puede ser debido a diferentes razones. En primer lugar, al temor por parte del obstetra al sangrado en general y particularmente al hematoma espinal vinculado a la anestesia. En este sentido, los estudios disponibles para estimar el riesgo de hemorragia en mujeres embarazadas bajo tromboprofilaxis farmacológica son limitados. La proporción de TEV fatal varía desde $0 \%$ a $1,91 \%$ con un promedio de $0,68 \%$ (IC 95\% 0,41\%-0,96\%), sin embargo no hay datos suficientes para estimar la tasa de sangrado fatal vinculado directamente a $\mathrm{HBPM}^{1}$. En un meta-análisis que combina los datos de 8 ensayos clínicos randomizados para evaluar el rol de la HBPM a dosis profilácticas en prevenir las complicaciones mediadas por la placenta, el riesgo de sangrado mayor durante el embarazo fue de $0,2 \%$ ( 1 de 470 ) y el riesgo de sangrado mayor en el post-parto fue de $0,6 \%(3 \text { de } 473)^{19}$. Otro meta-análisis publicado por Greer et al. evalúo la seguridad de la HBPM en 2.777 embarazos y observó que el sangrado mayor durante la gestación fue de $0,43 \%$ (95\% IC, $0,22 \%-0,75 \%$ ) y en el puerperio de $0,94 \%$ $(95 \% \text { IC, } 0,61 \%-1,37 \%)^{20}$. Por todo esto es que se debe realizar un adecuado balance riesgo/beneficio al momento de indicar la tromboprofilaxis. Nosotros consideramos que el riesgo trombótico de la realización de una cesárea de emergencia justificaría la realización de tromboprofilaxis farmacológica, y más en nuestro medio que no contamos con la posibilidad de realizar tromboprofilaxis mecánica con compresión neumática intermitente en forma sistemática en todas las cesáreas como lo sugerido por el ACOG.

Otro de los aspectos que en nuestro servicio explica la baja adherencia a la guía es que durante la implementación de la alerta escrita hemos detectado que la misma se coloca en un bajo número de historias clínicas (17,6\% de las mujeres). La utilización de un sistema de alerta que recuerde al médico la necesidad de realizar tromboprofilaxis ha sido demostrado en diferentes escenarios clínicos ${ }^{21-25}$. Asimismo, en una revisión sistemática 
y meta-análisis Cochrane se demuestra que las alertas incrementan la proporción de pacientes que reciben tromboprofilaxis en forma adecuada y disminuye la incidencia de eventos trombóticos ${ }^{26}$. En línea con estos trabajos hemos demostrado que las mujeres que se utilizó la alerta escrita presentaban en forma significativa mayor indicación y adherencia a la pauta de tromboprofilaxis $(60,9 \%)$ que aquellas en las que se omite colocar la alerta en la historia $(22,4 \%)$. Dado que no se han observado complicaciones trombóticas no hemos podido demostrar el impacto de la alerta en la disminución del número de eventos trombóticos.

Ha sido ampliamente documentado en la literatura el problema de baja adherencia a la tromboprofilaxis en diferentes escenarios clínicos y nuestro servicio no está ajeno a dicho proble$\mathrm{ma}^{27-31}$. Para mitigar esta baja adherencia a las guías se ha propuesto realizar instancias educativas al personal de salud vinculado a la asistencia de estas mujeres. Se está impulsando la inclusión universal de la alerta escrita en la historia clínica. Se está trabajando a nivel institucional para incorporar una alerta electrónica lo cual sin dudas mejorará la adherencia a la tromboprofilaxis.

En conclusión, en este estudio de corte transversal, hemos observado una baja adherencia a las guías de tromboprofilaxis del RCOG en mujeres sometidas a cesárea, particularmente en las mujeres que presentaban como único factor de riesgo la realización de una cesárea de emergencia. Hemos observado que en la práctica clínica contar con un sistema de alerta escrita mejora la adherencia del personal de salud a la tromboprofilaxis. Se continuará trabajando en busca de estrategias que mejoren la adherencia a la tromboprofilaxis en esta población.

\section{Referencias}

1. Kourlaba G, Relakis J, Kontodimas S, Holm MV, Maniadakis N. A systematic review and meta-analysis of the epidemiology and burden of venous thromboembolism among pregnant women. Int J Gynecol Obstet 2016; 132: 4-10. https://doi.org/10.1016/j.ijgo.2015.06.054.

2. Parunov LA, Soshitova NP, Ovanesov MV, Panteleev MA, Serebriyskiy II. Epidemiology of venous thromboembolism (VTE) associated with pregnancy. Birth Defects Res Part C Embryo Today Rev 2015; 105: 16784. https://doi.org/10.1002/bdrc.21105.

3. Say L, Chou D, Gemmill A, Tunçalp Ö, Moller A-B,
Daniels J, et al. Global causes of maternal death: a WHO systematic analysis. Lancet Glob Heal 2014; 2: e323-33. https://doi.org/10.1016/S2214-109X(14)70227-X.

4. Wik HS, Jacobsen AF, Sandvik L, Sandset PM. Prevalence and predictors for post-thrombotic syndrome 3 to 16 years after pregnancy-related venous thrombosis: a population-based, cross-sectional, case-control study. J Thromb Haemost 2012; 10: 840-7. https://doi. org/10.1111/j.1538-7836.2012.04690.x.

5. Rodger MA, Phillips $P$, Kahn SR, James AH, Konkle BA, PROSPER Investigators. Low-molecular-weight heparin to prevent postpartum venous thromboembolism. Thromb Haemost 2014; 113: 212-6. https://doi. org/10.1160/TH14-06-0485.

6. Gibbons L, Belizan JM, Lauer JA, Betran AP, Merialdi $M$, Althabe $F$. Inequities in the use of cesarean section deliveries in the world. Am J Obstet Gynecol 2012; 206: 331.e1-331.e19. https://doi.org/10.1016/j. ajog.2012.02.026.

7. Jacobsen AF, Skjeldestad FE, Sandset PM. Ante- and postnatal risk factors of venous thrombosis: a hospital-based case-control study. J Thromb Haemost 2008; 6: 905-12. https://doi.org/10.1111/j.1538-7836.2008.02961.x.

8. Blondon M, Casini A, Hoppe KK, Boehlen F, Righini M, Smith NL. Risks of Venous Thromboembolism After Cesarean Sections: A Meta-Analysis. Chest 2016; 150: 572-96. https://doi.org/10.1016/j.chest.2016.05.021.

9. Reducing the Risk of Venous Thromboembolism during Pregnancy and the Puerperium Green-top Guideline No. 37a, R. Coll. Obstet. Gynaecol. 2015. https://www. rcog.org.uk/globalassets/documents/guidelines/gtg-37a. pdf (accessed September 16, 2018).

10. Grille S, Vitureira G, Morán R, Retamosa L, Alonso V, Gómez LM, et al. Stevenazzi, Compliance with the 2009 Royal College of Obstetricians and Gynaecologists guidelines for venous thromboembolic disease prophylaxis in pregnancy and postpartum period in Uruguay. Blood Coagul Fibrinolysis 2018; 29: 252-6. https://doi. org/10.1097/MBC.0000000000000708.

11. Bonnar J. Can more be done in obstetric and gynecologic practice to reduce morbidity and mortality associated with venous thromboembolism? Am J Obstet Gynecol 1999; 180: 784-91. https://doi.org/10.1016/ s0002-9378(99)70648-4.

12. Clark SL, Belfort MA, Dildy GA, Herbst MA, Meyers JA, Hankins GD. Maternal death in the 21st century: causes, prevention, and relationship to cesarean delivery. Am J Obstet Gynecol 2008; 199: 36.e1-36.e5. https://doi. org/10.1016/j.ajog.2008.03.007.

13. Collins R, Scrimgeour A, Yusuf S, Peto R. Reduction in fatal pulmonary embolism and venous thrombosis by 
perioperative administration of subcutaneous heparin. Overview of results of randomized trials in general, orthopedic, and urologic surgery. N Engl J Med 1988; 318: 1162-73. https://doi.org/10.1056/NEJM198805053181805.

14. Collins R, Scrimgeour A, Yusuf S, Peto R. Reduction in Fatal Pulmonary Embolism and Venous Thrombosis by Perioperative Administration of Subcutaneous Heparin: Overview of Results of Randomized Trials in General, Orthopedic, and Urologic Surgery. J Urol 1989; 141: 218-9. https://doi.org/10.1016/s0022-5347(17)40703-8.

15. Mismetti P, Laporte S, Darmon JY, Buchmüller A, Decousus H. Meta-analysis of low molecular weight heparin in the prevention of venous thromboembolism in general surgery. Br J Surg 2001; 88: 913-30. https:// doi.org/10.1046/j.0007-1323.2001.01800.x.

16. Urbankova J, Quiroz R, Kucher N, Goldhaber SZ. Intermittent pneumatic compression and deep vein thrombosis prevention: A meta-analysis in postoperative patients. Thromb Haemost 2005; 94: 1181-5. https://doi. org/10.1160/TH05-04-0222.

17. James A. The American Congress of Obstetricians and Gynecologists (ACOG) Committee of Practice Bulletins: obstetrics practice bulletin \#138 inherited thrombophilias in pregnancy. Obstet Gynecol 2013; 122: 706-17. https://doi.org/10.1097/AOG.0b013e3182310c4c.

18. Bates SM, Greer IA, Middeldorp S, Veenstra DL, Prabulos AM, Vandvik PO, VTE. Thrombophilia, Antithrombotic Therapy, and Pregnancy. Chest 2012; 141: e691S-e736S. https://doi.org/10.1378/chest.11-2300.

19. Rodger MA, Gris J-C, JIP de Vries, Martinelli I, Rey É, Schleussner E, et al. Low-Molecular-Weight Heparin for Placenta-Mediated Pregnancy Complications Study Group, Low-molecular-weight heparin and recurrent placenta-mediated pregnancy complications: a meta-analysis of individual patient data from randomised controlled trials. Lancet 2016; 388: 2629-41. https://doi. org/10.1016/S0140-6736(16)31139-4.

20. Greer IA, Nelson-Piercy C. Low-molecular-weight heparins for thromboprophylaxis and treatment of venous thromboembolism in pregnancy: a systematic review of safety and efficacy. Blood 2005; 106: 401-7. https://doi. org/10.1182/blood-2005-02-0626.

21. Figueroa R, Alfonso A, López-Picazo J, Gil-Bazo I, García-Mouriz A, Hermida J, et al. Improvement of appropriate pharmacological prophylaxis in hospitalised cancer patients with a multiscreen e-alert system: a single-centre experience. Clin Transl Oncol 2019; 21: 805-9. https://doi.org/10.1007/s12094-018-1986-1.

22. Lecumberri R, Panizo E, Gómez-Guiu A, Varea S, García-Quetglas E, Serrano M. Economic impact of an electronic alert system to prevent venous thromboembolism in hospitalised patients. J Thromb Haemost 2011; 9: 110815. https://doi.org/10.1111/j.1538-7836.2011.04282.x.

23. Woller SC, Stevens SM, Evans RS, Wray D, Christensen J, Aston VT, et al. Electronic alerts, comparative practitioner metrics, and education improve thromboprophylaxis and reduce venous thrombosis in community hospitals. Res Pract Thromb Haemost 2018; 2: 481-9. https://doi.org/10.1002/rth2.12119.

24. Spirk D, Stuck AK, Hager A, Engelberger RP, Aujesky D, Kucher N. Electronic alert system for improving appropriate thromboprophylaxis in hospitalized medical patients: a randomized controlled trial. J Thromb Haemost 2017; 15: 2138-46. https://doi.org/10.1111/ jth.13812.

25. Woller SC, Stevens SM, Evans RS, Wray DG, Christensen JC, Aston VT, et al. Electronic Alerts, Comparative Practitioner Metrics, and Education Improves Thromboprophylaxis and Reduces Thrombosis. Am J Med 2016; 129: 1124.e17-1124.e26. https://doi.org/10.1016/j. amjmed.2016.05.014.

26. Kahn SR, Diendéré G, Morrison DR, Piché A, Filion $\mathrm{KB}$, Klil-Drori AJ, et al. Effectiveness of interventions for the implementation of thromboprophylaxis in hospitalised patients at risk of venous thromboembolism: An updated abridged Cochrane systematic review and meta-analysis of randomised controlled trials. BMJ Open 2019; 9: e024444. https://doi.org/10.1136/bmjopen-2018-024444.

27. Amin A, Stemkowski S, Lin J, Yang G. Thromboprophylaxis rates in US medical centers: success or failure? J Thromb Haemost 2007; 5: 1610-6. https://doi.org/10.1111/j.1538-7836.2007.02650.x.

28. Cohen AT, Tapson VF, Bergmann J-F, Goldhaber SZ, Kakkar AK, Deslandes B, et al. Venous thromboembolism risk and prophylaxis in the acute hospital care setting (ENDORSE study): a multinational cross-sectional study. Lancet (London, England) 2008; 371: 387-94. https://doi.org/10.1016/S0140-6736(08)60202-0.

29. Clark BM, d'Ancona G, Kinirons M, Hunt BJ, Hopper A. Effective quality improvement of thromboprophylaxis in acute medicine. BMJ Qual Saf 2011; 20: 460-4. https://doi.org/10.1136/bmjqs.2010.044503.

30. Thavarajah D, Wetherill M. Implementing NICE guidelines on risk assessment for venous thromboembolism: failure, success and controversy. Int J Health Care Qual Assur 2012; 25: 618-24. https://doi. org/10.1108/09526861211261217.

31. Byrne S, Weaver DT. Review of thromboembolic prophylaxis in patients attending Cork University Hospital. Int J Clin Pharm 2013; 35: 439-46. https://doi. org/10.1007/s11096-013-9760-5. 